NISTIR 7192

\title{
2004 Conference on IEEE 1588, Standard for a Precision Clock Synchronization Protocol for Networked Measurement and Control Systems
}

Kang B. Lee

John C. Eidson 



\title{
2004 Conference on IEEE 1588, Standard for a Precision Clock Synchronization Protocol for Networked Measurement and Control Systems
}

\author{
Kang B. Lee \\ Manufacturing Metrology Division \\ Manufacturing Engineering Laboratory \\ John C. Eidson \\ Agilent Technologies
}

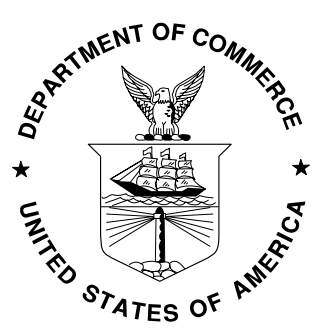

U.S. DEPARTMENT OF COMMERCE

Donald L. Evans, Secretary

TECHNOLOGY ADMINISTRATION

Phillip J. Bond, Under Secretary of Commerce for Technology NATIONAL INSTITUTE OF STANDARDS AND TECHNOLOGY 


\title{
2004 Conference on IEEE 1588, Standard for a Precision Clock Synchronization Protocol for Networked Measurement and Control Systems
}

\author{
Co-sponsored by \\ NIST and IEEE Instrumentation and \\ Measurement Society
}

\author{
NIST \\ Gaithersburg, Maryland \\ September 27-29, 2004 \\ Conference Sessions: Lecture Room A \\ Plug-fest Demonstration: Lecture Room B
}

\section{Disclaimer}

Certain commercial equipment, instruments, or materials are identified in this proceedings to specify the experimental procedure adequately. Such identification is not intended to imply recommendation or endorsement by the National Institute of Standards and Technology, nor is it intended to imply that the materials or equipment identified are necessarily the best available for the purpose. 


\section{TABLE OF CONTENTS}

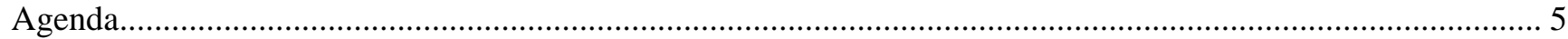

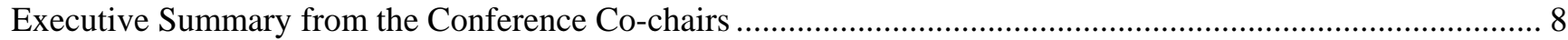

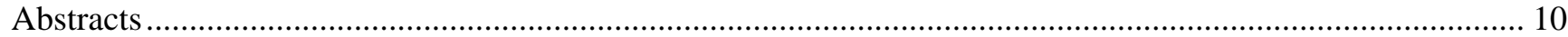

Technical Presentations- Session I

A Flexible and Scalable Network Simulation Environment for Clock Synchronization-

Roland Höller, Georg Gaderer, Hannes Muhr, Nikolaus Kerö,

Vienna University of Technology and Oregano Systems

Implementation and Performance of Time Stamping Techniques-

Hans Weibel, Dominic Béchaz, Zurich University of Applied Sciences ...................................................................... 24

Industrial Automation Requires Synchronization of Line Topology- Antonius Boller, Siemens AG ................................. 39

\section{Technical Presentations- Session II}

IEEE 1588 Ethernet Switch Transparency- No Need for Boundary Clocks!-

Sven Nylund, Oyvind Holmeide, OnTime Networks AS

Bridging Networks with PTP-

Karl Weber, Jürgen Jasperneite, Siemens AG and Phoenix Contact GmbH ........................................................ 56

Implementation Results of an IEEE 1588 Boundary Clock- Dirk Mohl, Hirschmann Electronics ...................................... 65

PHYs and Symmetrical Propagation Delay- Thomas Müller, Alexander Ockert, Hans Weibel,

Zurich University of Applied Sciences and Hilscher . 78

STANDARDS AND BuSINESS ACTIVITIES

Report of the IEEE 1588 Task Force on User Requirements- Silvana Rodrigues, Steve Zuponcic,

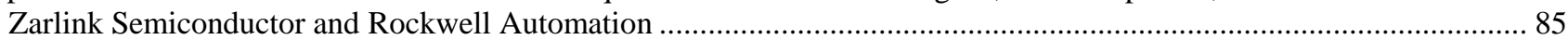

Report of the IEEE 1588 Task Force on Technical Extension- John C. Eidson, Agilent .................................................. 90

Report of the Conformance Task Group- Oyvind Holmeide, OnTime Networks AS .......................................................... 95

A Proposal to Open a PAR Based on the Work of the IEEE 1588 Task Groups-

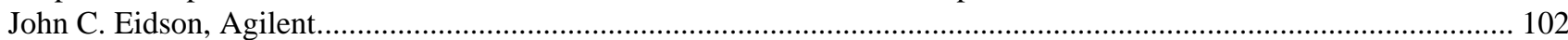

A Proposal to Create a Trade Association to Promote IEEE 1588 Technology- John Eidson, Agilent .............................. 108

Plug Fest Report- Anatoly Moldovansky, Rockwell Automation............................................................................... 113

\section{Technical Presentations- Session III}

IEEE 1588 over IEEE 802.11b for Synchronization of Wireless Local Area Network Nodes-

Afshaneh Pakdaman, Todor Cooklev, John Eidson, San Francisco University and Agilent.....

High Accuracy Clock Synchronization Using IEEE 1588-

Pritam Baruah, Jeff Burch, Pruthvi Chaudhari, Paul Corredoura, John Eidson, Andrew Fernandez

Bruce Hamilton, John Stratton, Dieter Vook, Agilent

Primary Timing Reference Sources for IEEE 1588 Systems- Paul Myers, Spectracom ................................................... 137

Technical Presentations- Session IV

DeviceNet Adaptation of IEEE 1588- Ron Holl, Dave VanGompel, Rockwell Automation ................................................ 149

Hardware Assisted IEEE 1588* Implementation in a Next Generation Intel Network Processor-

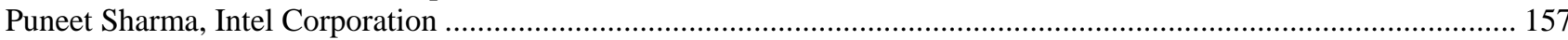

Interfacing Mil Standard Equipment to an IEEE 1588 Enabled Ethernet Network-

John MacKay, Progeny Systems

Automatic Test Systems using LAN-based Synthetic Instruments and the role of IEEE1588-

John Stratton, Agilent.

IEEE 1588 in Telecommunications Applications- Dave Tonks, Semtech .................................................................. 184

Telecom Update to IEEE 1588 Workshop- Glenn Algie, Nortel Networks.................................................................... 190

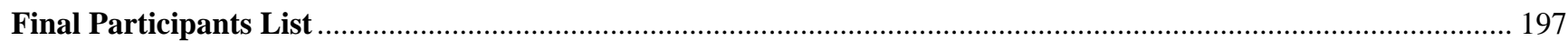




\section{AGENDA}

\section{Monday, September 27, 2004}

- $\quad$ 8:30-9:00 AM: Meeting of Plug-fest participants. This session is open only to Plug-fest participants.

- 9:00 AM - 12:15 PM: Plug-fest integration tests. This session is open only to Plug-fest participants.

- 10:30 AM: Morning coffee break

- 12:15-1:15 PM: Lunch at NIST cafeteria (not included in registration fee)

- 1:15-5:00 PM: Plug-fest integration tests. This session is open only to Plug-fest participants.

- 12:30-1:15 PM: Tutorial participants registration

- 1:15-5:00 PM: IEEE 1588 Tutorial: John C. Eidson, Agilent Technologies

- 3:00 PM: Afternoon refreshment break

\section{Tuesday, September 28, 2004}

- 8:00 AM: Bus leaves conference hotel for NIST facility

- 8:30-9:00 AM: Continental breakfast, meet other attendees, pick up conference badges and material. (Allow 30 minutes from arrival at the main gate due to security and parking)

- 9:00-9:15 AM: Conference Opening

Moderator: Kang Lee, NIST

o Welcome from Dr. Hratch G. Semerjian - Acting Director of NIST

o Administrative details

- 9:15 AM to 10:30 AM: Technical Paper Presentations Session I

Moderator: Oyvind Holmeide, OnTime Networks AS

o 9:15-9:40 AM: A Flexible and Scalable Network Simulation Environment for Clock Synchronization: Roland Hoeller, Georg Gaderer, Hannes Muhr, Nikolaus Kero, Vienna University of Technology and Oregano Systems

o 9:40-10:05 AM: Implementation Design and Performance Issues: Hans Weibel, Dominic Béchaz, Zurich University of Applied Science

o 10:05-10:30 AM: Industrial Automation Requires Synchronization of Line Topology: Antonius Boller, Siemens

- $\quad$ 10:30 - 10:50 AM: Morning coffee break

- 10:50 AM-12:30 PM: Technical Paper Presentations Session II

Moderator: John C. Eidson, Agilent Technologies

o 10:50-11:15 AM: IEEE 1588 Ethernet Switch Transparency: Sven Nylund, Oyvind Holmeide, OnTime Networks AS

o 11:15-11:40 AM: Bridging Networks with PTP: Karl Weber, Siemens, Jürgen Jasperneite, Phoenix Contact GmbH

o 11:40 AM-12:05 PM: Implementation Results of an IEEE 1588 Boundary Clock: Dirk S. Mohl, Hirschmann Electronics

o 12:05-12:30 PM: PHYs and Symmetrical Propagation Delay: Thomas Müller, Zurich University of Applied Science, Alexander Ockert, Hilscher, Hans Weibel, Zurich University of Applied Science

- 12:30-1:30 PM: Lunch at NIST cafeteria 
o 1:30-1:50 PM: Report of the User Requirements Task Group: Silvana Rodrigues, Zarlink Semiconductor, Steve Zuponcic, Rockwell Automation

o 1:50-2:10 PM: Report of the Technical Extensions Task Group: John C. Eidson, Agilent Technologies

o 2:10-2:30 PM: Report of the Conformance Task Group: Oyvind Holmeide, OnTime Networks AS

o 2:30-2:50 PM: Presentation of Draft PAR: John C. Eidson, Agilent Technologies

o 2:50-3:10 PM: Proposal for IEEE 1588 Trade Association: John C. Eidson, Agilent Technologies

- 3:10-3:30 PM: Plug-fest Introduction: Objectives, Participants, and Results

Moderator: Anatoly Moldovansky, Rockwell Automation

- 3:30-3:45 PM: Afternoon refreshment break

- $\quad$ 3:30-5:00 PM: Attendees view and discuss Plug-fest in Lecture Room B

- 5:15 PM: Bus leaves NIST for conference hotel

\section{- 6:30-9:00 PM: Conference Reception And Dinner}

Bus leave conference hotel restaurant

o 6:45- 7:15 PM: No-host cash bar

o 7:15 PM: Conference Dinner

o 9:00 PM: Bus leave restaurant for conference hotel

\section{Wednesday, September 29, 2004}

- 8:00 AM: Bus leaves conference hotel for NIST facility

- 8:30-9:00 AM: Continental breakfast

- 9:00 AM to 10:15 AM: Technical Paper Presentations Session III

Moderator: John D. McKay, Progeny Systems

o 9:00-9:25 AM: IEEE 1588 over IEEE 802.11b for Synchronization of Wireless Local Area Network Nodes: Afshaneh Pakdaman, Todor Cooklev, San Francisco State University; John Eidson, Agilent Technologies

o 9:25-9:50 AM: High Accuracy Clock Synchronization Using IEEE 1588: Pritam Baruah, Pruthvi Chaudhari, Paul Corredoura, John C. Eidson, Andrew Fernandez, Bruce Hamilton, John Stratton, Dieter Vook, Agilent Technologies

o 9:50-10:15 AM: Primary Timing Reference Sources for IEEE-1588 Systems: Paul Myers, Spectracom

- 10:15 - 10:35 AM: Morning coffee break

- 10:35 AM to 12:15 PM: Technical Paper Presentations Session IV

Moderator: Anatoly Moldovansky, Rockwell Automation

o 10:35-11:00 AM: DeviceNet Adaptation of IEEE 1588: Ron Holl, Dave VanGompel: Rockwell Automation

o 11:00-11:25 AM: Hardware Assisted IEEE1588 Implementation in a Next Generation Intel Network Processor: Puneet Sharma, Intel Corporation

o 11:25-11:50 AM: Interfacing Mil Standard Equipment to an IEEE 1588 Enabled Ethernet Network: John D. MacKay, Progeny Systems

o 11:50 AM-12:15 PM: Automatic Test Systems using LAN-based Synthetic Instruments and the Role of IEEE 1588: John Stratton, John Swanstrom, Agilent Technologies 
- 12:15-1:15 PM: Lunch at NIST cafeteria

- 1:15 to 2:05 PM: Technical Paper Presentations Session V

Moderator: John C. Eidson, Agilent Technologies

o 1:15-1:40 PM: IEEE 1588 in Telecommunication Applications: Dave Tonks, Semtech

o 1:40-2:05 PM: IEEE 1588 Telecom Use Cases and L2 Ethernet Multicast: Glenn Algie, Nortel Networks

- 2:05-3:50 PM: Discussion Session

Moderator: Kang Lee, NIST \& John Eidson, Agilent Technologies

o 2:05-2:30 PM: Discussion \& Attendee Feedback on IEEE 1588 PAR

o 2:30-3:00 PM: Discussion \& Attendee Feedback on IEEE 1588 Trade Association

- 3:00-3:20 PM: Afternoon refreshment break \& networking

o 3:20-3:50 PM: Open Discussion on Other Issues

- 3:50-4:00 PM: Closing Comments

o Kang Lee \& John Eidson

- 4:00 PM: Conference adjournment

- 4:30 PM: Bus leaves NIST for conference hotel 


\section{EXECUTIVE SUMMARY FROM THE CONFERENCE CO-CHAIRS}

The conference was hosted by NIST on September 27-29, 2004 and was cosponsored by the Institute of Electrical and Electronics Engineers (IEEE) Instrumentation and Measurement Society. Acting Director of the National Institute of Standards and Technology (NIST), Dr. Hratch Semerjian, opened the conference with a warm welcome. Dr. Semerjian spoke of the importance of standards on components and system interoperability and his assertion of interoperability's role in the expansion of the PC market to its grand scale today. Dr. Semerjian described how standards are basic to the culture of NIST. Pursuing device and system interoperability based on standards is one of NIST's goals. More than seventy attendees participated in the conference, coming from diverse areas such as instrumentation and measurement, industrial automation, aerospace, power generation, semiconductor manufacturing, and telecommunication.

The three-day event began with a tutorial on the IEEE 1588 standard. The tutorial was unexpectedly well attended by more than sixty percent of the attendees. The main conference started on the second day. The interoperability of components and devices was demonstrated by seven companies and a university in an afternoon session informally dubbed the "Plug-fest." The devices were built to IEEE 1588 specifications, and showed that they could be synchronized to a master clock to sub-microsecond accuracy. The interoperability demo was led by Anatoly Moldovansky of Rockwell Automation, with participation from Agilent Technologies, Hirschmann Electronics, OnTime Networks AS, Rockwell Automation, Semtech Corp, Siemens, and Zurich University of Applied Sciences.

Participants were impressed with the smoothness and outcome of the interoperability demonstrations. Some components and systems were able to achieve clock synchronization to within $+/-40$ nanoseconds based on a master clock signal from a global positioning system (GPS) antenna located on the lawn outside the conference facility. This illustrated the effectiveness of the 1588 standard and the ease with which devices can be built to its specifications.

The technical sessions covered subjects such as: primary timing reference sources for IEEE 1588 systems; high accuracy clock synchronization down to a nanosecond for precision measurements; network simulation environment for clock synchronization; device and microchip requirements; and adaptation, implementation, and application of IEEE 1588 in industrial automation, military, and telecommunications. A presentation by a graduate student on the application of IEEE 1588 for the synchronization of wireless local area network nodes based on $802.11 \mathrm{~b}$ created quite a discussion. The field of wireless communications is of great interest to the attendees, who expressed a wish to see more detailed results in this area at the next conference.

As a result of the 2003 IEEE 1588 Workshop, three task groups were formed to address the issues of user requirements, technical extensions, and conformance of the IEEE 1588 Standard. The results of these three task groups were presented at the conference. These results were reflected in the presentation of a potential draft project authorization request (PAR) to IEEE and the possibility of forming an IEEE 1588 user group or trade association. An open forum was held on the last day of the conference to further discuss the issues of creating a PAR to revise the IEEE Std 1588-2002 Standard and the formation of a user or trade group to promote the standard and facilitate interoperability tests, and of enhancing the standard to expand its coverage from the instrumentation and measurement to other industries such as industrial automation and telecommunications.

Based on the feedbacks of the attendees, there was overwhelming consensus to reopen the IEEE 1588 standard to include:

1. Resolution of known errors,

2. Conformance enhancements,

3. Enhancements for increased resolution and accuracy,

4. Improvements to system management capability,

5. Mapping to DeviceNet,

6. Modifications for variable Ethernet headers (Annex D),

7. Prevention of error accumulation in cascaded topologies,

8. Mapping to Ethernet layer-2 small frame, shorter sync_interval,

9. Extensions to enable implementation of redundant systems, and

10. Improvements to extension mechanism.

If the standard is reopened some attendees suggested that the following additional items be considered as part of the scope: 
- Alignment of IEEE 1588 and NTP stratum,

- Clock ID (identification) alignment with telecom T1.101 G.812,

- Security considerations- currently it is possible to take over the GR Role, e.g. with preferred master" or to manipulate sync packets, security IPv6 should be considered for backward compatibility, and there is a need to scope the problem and begin to get around it,

- Internet protocol version 6 (IPv6) Authentication - any IPv6 issues beyond Ethernet header size,

- Authentification for network security, IPv6 is essential or we will development a proprietary protocol version by necessity, or layer-2 mapping,

- Backward compatibility with current standard as its defined today,

- IEEE 1451 TEDS (transducer electronic data sheets)-like information on clock parameters. Better ID of stratum and source,

- Main point is authentication security. Other protocols do this at layer-3. However, authentification signature is large and it grows over time. Further, modification of packets violates authentication signatures or they need to be recalculated. At layer-3 IEEE 1588 should not do its own security. This is redundant and risky.

- Metadata format to allow description of oscillator and time source-ups receiver, vendor extensions for management information (MIB) for the simple network management protocol (SNMP), vendor extensions for metadata,

- Shouter frames and variable sync internal with unicort option will be vital to wireless sensor nets,

- Best master group,

- Some goals may be harder to achieve than others. Consider formulating more than one PAR,

- Inclusion of sync or management messages that permit a grand master to discover the time error of each slave. A message that can be decoded to produce a hardware interrupt to allow a MC (master clock) in a computer without a real-time OS (operating system) to execute an emergency operation in real time.

- Mapping to the control area network (CAN) open, and

- SNMP as a requirement for system management.

\section{Conference Proceedings}

The proceedings for the conference will be published as a NISTIR (internal report) before distribution. They will be posted in the IEEE 1588 website at http://ieee1588.nist.gov as soon as it is approved for publication by NIST.

\section{Future Workshop}

There was brief discussion on the plan for a future IEEE 1588 conference. Most attendees wanted to have another one. Location will be either at NIST or in Europe, which is to be determined. More detailed plans for the next workshop will be presented in the spring of 2005. 


\title{
$\underline{\text { ABSTRACTS }}$
}

Authors Company

Ron Holl, Dave VanGompel
Rockwell

Automation

\section{DeviceNet Adaptation of IEEE-1588}

DeviceNet is an extremely popular device level industrial network and is ideal for many applications requiring synchronized time, yet there is no current mechanism to accomplish this. This paper describes how DeviceNet will provide this function by adapting it to IEEE-1588 as a standardized PTP network technology. The adaptation includes selection of a message timestamp point, specification of the UUID, definition of both the PTP message format and PTP addressing on the subnet, and integration into the DeviceNet architecture.

\begin{tabular}{ll}
\hline $\begin{array}{l}\text { Roland Hoeller, } \\
\text { Georg Gaderer, } \\
\text { Hannes Muhr; }\end{array}$ & $\begin{array}{l}\text { Vienna } \\
\text { University of } \\
\text { Technology }\end{array}$ \\
Nikolaus Kero & $\begin{array}{l}\text { Oregano } \\
\text { Systems }\end{array}$
\end{tabular}

\section{A Flexible and Scalable Network Simulation Environment for Clock Synchronization}

The problem of synchronization of clocks in distributed systems has received much scientific attention throughout the last decades. A variety of algorithms has been published and issues like fault tolerance or achievable accuracy have been addressed. Nevertheless most applications found themselves sufficiently well synchronized by using means like the Network Time Protocol (NTP) or the Global Positioning System (GPS). Not only the recent interest in using Ethernet for industrial automation or even in sensor networks, but also the advent of the Institute of Electrical and Electronics Engineer's (IEEE) Standard for a Precision Clock Synchronization Protocol for Networked Measurement and Control Systems (IEEE 1588) let high accuracy clock synchronization be a new discussed under the light of new applications and technical constraints.

This paper presents a flexible and scalable network simulation environment, which allows for detailed and fast investigation of the major parameters of clock synchronization for any given network technology or topology. The simulation environment's architecture will be presented and simulation results together with their possible influences on existing technology or standards will be discussed.

\author{
Pritam Baruah, \\ Pruthvi Chaudhari, \\ Agilent \\ Paul Corredoura, John \\ Technologies \\ C. Eidson, Andrew \\ Fernandez, \\ Bruce Hamilton, John \\ Stratton, \\ Dieter Vook
}

\section{High Accuracy Clock Synchronization using IEEE 1588}

There exist applications in the field of measurement instrumentation, military systems, and telecommunications with synchronization accuracy specifications extending to the nanosecond or sub-nanosecond range. This paper discusses the practical difficulties in achieving this level of synchronization and proposes extensions to IEEE 1588 to make this possible. Experimental results on prototype implementations will be discussed. 


\section{Afshaneh Pakdaman, Todor Cooklev;}

John Eidson
San Francisco

State University

Agilent

Technologies

\section{IEEE 1588 over IEEE 802.11b for synchronization of wireless local area network nodes}

IEEE 1588 is a new standard to synchronize independent clocks running on separate nodes of a distributed measurement and control system to high degree of accuracy. It used a precision-time protocol (PTP). In this paper it is advanced a method to implement clock synchronization over IEEE 802.11b Wireless LAN (WLAN). Practical experiments are presented. One conclusion is that IEEE 1588 can be implemented over 802.11b with an accuracy of 400ns.

\begin{tabular}{ll}
\hline Puneet Sharma & $\begin{array}{l}\text { Intel } \\
\text { Corporation }\end{array}$
\end{tabular}

\section{Hardware Assisted IEEE1588 Implementation in a Next Generation Intel Network Processor}

This paper will describe the hardware-assisted IEEE1588 implementation in a future planned Intel ${ }^{\circledR}$ network processor. A brief overview of the IEEE1588 standard is provided, with particular emphasis on Ethernet applications. The general pros and cons of purely hardware vs. software-oriented IEEE1588 implementations are also discussed and applied to Intel's co-hardware and software IEEE1588 implementation. A detailed description of the IEEE 1588 hardware logic and the Intel XScale ${ }^{\circledR}$ core-based software programming model is included. Finally, some examples of targeted industrial applications for IEEE1588 are described.

Note regarding the 20-minute paper presentation at the Conference: The 20-minute presentation will NOT include a brief overview of the IEEE1588 standard as attendees should be familiar with the standard.

\begin{tabular}{ll}
\hline Hans Weibel & Zurich \\
& University of \\
& Applied Science
\end{tabular}

\section{Implementation design and performance issues}

There exist applications where independence of specialized hardware is more important than accuracy. The Zurich University of Applied Sciences has evaluated the performance of software based time stamping methods. The test setup consists of an IEEE 1588 implementation which is capable to deliver three time stamps per transmission/reception of time critical messages simultaneously: The first time stamp is taken by hardware at the MII, the second at the entry point of the network interface driver's interrupt service routine and the third one is delivered by PCAP. A comparison of the time stamps allows the performance of different methods to be evaluated. The PTP protocol engine is able to select one of the three available time stamps as the source to calculate offset and delay. The synchronization behaviors and accuracy of different configurations can be analyzed. An interesting configuration is a hardware based master clock (e.g. a boundary clock located in a switch) combined with purely software based slave clocks.

Antonius Boller
Siemens

\section{Industrial automation requires synchronization of line topology}

The high data transfer rate attainable through the Ethernet's physical properties opens new dimensions for real-time applications.

A future-oriented concept must have at its core a wide, generally accepted basis, and must be expandable. Seen from this point of view, there is no alternative to switching technology. The advantages of higher data transfer rates, full-duplex communication and collision-free access, however, are bought at the expense of 
an unsymmetrical communication. Due to the fact that frames are buffered in the switch and the length of time they remain there depends on the network load this may even lead to frame loss in critical situations. This is a most unfortunate peculiarity in the case of real-time applications and the reason why it is not possible to fulfil the requirements of the switched-Ethernet-based motion control applications with the existing IEEE 1588 method. Especially in the industrial automation a basic requirement of the network is the ability for line topology. Hence a cascade of switches has to be synchronized. With today's IEEE 1588-method the required accuracy for a line of switches causes problems.

The presentation shows the need for real time Ethernet in the industrial automation and the problems when using IEEE 1588 for synchronization issues in this area. Furthermore it shows a method which solves these problems.

John D. MacKay

\section{Interfacing Mil Standard Equipment to an IEEE 1588 Enabled Ethernet Network}

This paper will discuss the unique requirements for interfacing a number of military standard devices to a 1588 network. The current trend for signal processing on military platforms is to maximize the usage of Commercial Off the Shelf (COTS) products rather than developing full mil-grade equipment. This approach provides a great deal of advantage to the system developer, because it provides access to a variety of low cost high performance devices that have a great deal of field experience and customer support. The drawbacks to this approach occur at the edges of the system, where these COTS devices must interface either with very specialized sensor and transducer equipment, or with 'legacy' standard busses and protocols.

A COTS system with Ethernet as the core network can be subject to this issue, but this can be compounded by the need to provide highly accurate timestamp information via 1588. Typically the legacy devices are clocked by their own internal timing, and drive the system time via COTS interface cards. Timestamps therefore occurred at the 'front end' of the process string, and became embedded in the data stream. While the insertion of timestamp data in a 1588-enabled system would likely be the same, the source of time would need to be either a 1588 network device or a legacy device modified to be 1588enabled. There are issues with both of these solutions.

These options will be discussed for this use case as well as others. A use case that would require the 1588 protocol to be implemented on a mil-standard asynchronous bus will also be discussed.

Dirk S. Mohl Hirschmann
Electronics

\section{Implementation Results of an IEEE 1588 Boundary Clock}

Boundary clocks are necessary to distribute the precise time over network components like switches and routers. To build such a boundary clock inside an Ethernet switch beside the standard functionality several additional points have to be taken into account.

Ethernet Switches typically use SNMP as management protocol, so relevant parts of the IEEE1588 managed objects have to be accessible through SNMP. Also a switch often gets or provides time over SNTP. The question now is how to combine these two protocols and not to loose IEEE1588 precision. The more IEEE 1588 Switches are used the network the more the issue of cascading Boundary Clocks and its effect on precision and system startup has to be analyzed. 
The necessity of cascading boundary clocks often is imposed from the network topology of the application. One aspect of using IEEE1588 is derived from Industrial Automation Technology. There IAONA, the independent platform organization for Industrial Ethernet may give ideas about usage, applications and topologies of the network.

\begin{tabular}{ll}
\hline Sven Nylund & OnTime \\
Oyvind Holmeide & Networks AS
\end{tabular}

\section{IEEE 1588 Ethernet switch transparency- No need for Boundary Clocks!}

One of the main IEEE1588 properties is related to the handling of variable network latency between the Grand Master clock and the Slave clocks. I.e. the network load dependable latency through the network elements (e.g. Ethernet switches). This is handled if IEEE1588 Boundary clocks are used on the network path between Grand Master and the Slave clocks. However, this means that each network element in the network must support full Master, Slave and the Best Master Algorithm with increased complexity and cost as the result.

A simpler and cheaper approach is based on using network elements with IEEE1588 transparency and still achieves the same level of timing accuracy on the Slave clocks and being compliant with the IEEE1588 standard. This paper describes the principles of a network element with IEEE1588 transparency.

\begin{tabular}{|c|c|c|}
\hline Karl Weber & Siemens & Bridging Networks with PTP \\
\hline & & $\begin{array}{l}\text { Discuss influence of switches in networking today and how it correlates to } \\
\text { 1588. General procedures running at switches and infrastructure of such } \\
\text { switches. Discuss need for time synchronization in switches Propose } \\
\text { Architecture for "PTP Bridges" that enhance accuracy and reduce resource } \\
\text { utilization in switches. Criteria for a PTP Bridge protocol that will be accepted } \\
\text { by most switch manufacturer }\end{array}$ \\
\hline
\end{tabular}

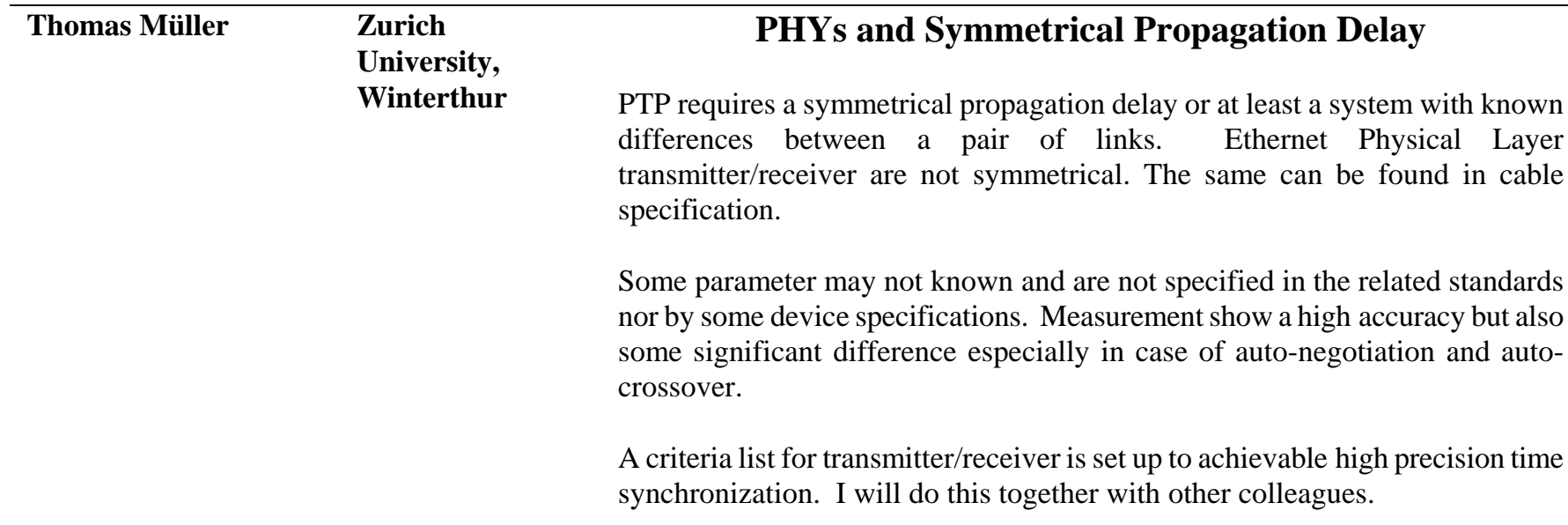

Dave Tonks

\section{IEEE 1588 in Telecommunication Applications}

Advanced

Communications

Division, Southampton UK
IEEE 1588 is being considered for various applications within telecommunication networks, including delivering a common time base across a network for billing purposes and for synchronizing service points which have become isolated by use of a packet network. This paper investigates a few of these applications and discusses a number of issues, including expected limitations on network topology, and known or likely performance goals, and, most importantly, probable barriers to adoption. The paper goes on to discuss 
how these issues could be tackled, and in particular how the current IEEE 1588 standard could be adapted to simplify its adoption in telecommunication applications.

The migration of telecommunication networks away from their traditional circuit-based architecture and towards an all-encompassing packet network has begun. The principal drivers behind this are significant cost-reductions in both capex and opex, and simpler roll-out of new services. When completed, it will be seen to have been a massive overhaul of networking technology, covering all aspects of the network, from switching and transmission to operational, administration and maintenance activities. However, many of the services which have been enjoyed for many years will continue to exist and these are not well served by packet networks. They have critical time dependencies which, if not satisfied, will cause the service to fail to maintain the high levels of customer satisfaction they enjoy today. The problem, then, is in finding ways to satisfy the critical time dependencies in a packet network. IEEE 1588 offers a cost-effective way to deliver timing in packet networks, providing certain limitations can be overcome.

Packet networks differ considerably from traditional circuit-switched networks. The possibility of departing from the 'fixed' route per call, and the use of service level agreements in which it is necessary to know not only just how much traffic of each particular type was delivered, but also how much of it met the delay targets, means that network operations such as traffic-counting have to be moved to the edge of the network. This demands that accurate time be made available right at the edge of the network. IEEE 1588 can provide that time.

Carrying time-dependent services in a packet network is often done using a circuit-emulation technique. But this is best done when a common clock is available at both ends of the connection. Traditional networks inherently provide this clock but packet networks cannot. Adaptive clocking techniques are available but suffer from network behaviors and can only offer a lower performance. Customer complaints could be common. Alternatively, IEEE 1588 can provide the common clock at the ends of the connection and so help maintain quality of delivery. This paper explores these, and other, applications.

\section{John Stratton, John Swanstrom, \\ Agilent
Technologies}

\section{Automatic Test Systems using LAN-based Synthetic Instruments and the Role of IEEE 1588}

With the current trend to drive down the total cost of ownership of Automatic Test Systems (ATS), industry standard open architectures have been seen as both a way of driving down the cost of test (for design and manufacturing) and reducing the size of the ATS platforms (eliminate the redundant hardware).

A LAN-based synthetic instrument architecture offers an alternative to the traditional approach that allows systems integrators and manufacturers to minimize the total cost of ownership from digital to the highest performance millimeter wave applications. IEEE 1588 is proposed as the long-term solution for synchronization is the LAN environment.

This paper will analyze the current trends in computer architectures and how it is used in synthetic instrument based ATSs. It will show how future trends in component technologies will drive how synthetic instruments might be designed. And finally, it will show how customers and instrument providers can quickly implement current capability and next generation test technology. 
This presentation reviews and compares Primary Time Reference sources for IEEE 1588 systems. Primary Time Reference sources such as GPS, Loran and cellular GSM/CDMA are reviewed. The technical merits, characteristics, performance differences, costs applications and availability are contrasted. The use of these primary time references in the design of a grand master clock and resulting predicted precision of 1PPS outputs is examined. The application of various oscillator types to IEEE-1588 systems is compared in light of primary timing reference selection, and holdover performance. 


\section{FINAL PARTICIPANTS LIST}

\author{
Mr. Glenn Algie \\ Nortel Networks \\ 3500 Carling Ave \\ Ottawa, K2H 8E9 \\ CANADA \\ 613-765-3425 \\ algie@nortelnetworks.com \\ Anthony Ander \\ Agilent Technologies \\ Penang, 10460 \\ MALAYSIA \\ 604-680-7231 \\ 604-645-6604

\section{Galina Antonova} \\ 8525 Baxter Pl., Ste 100 \\ Burnaby, BC V5A 4V7 \\ CANADA \\ 604-421-8629 \\ 604-421-8707 \\ galina.antonova@ge.com

\section{Dr. Douglas Arnold} \\ Symmetricom \\ 3750 Westwind Blvd \\ Santa Rosa, CA 95403 \\ USA \\ 707-636-1929, Fax: 707-527-6640 \\ darnold@symmetricom.com

\section{A. Baller} \\ Siemens AG \\ Gleiwitzer Str 555 \\ GERMANY

\section{Mr. Nick Barendt} \\ VXI Technology, Inc. \\ 5425 Warner Rd, Ste 13 \\ Valley View, OH 44125 \\ USA \\ 216-447-4059, fax: 216-447-8951 \\ nbarendt@vxitech.com \\ Mr. Dominic Bechaz \\ Zurich Univ. of Applied Sciences \\ Technikumstrasse 9 \\ Postfach 805, Winterthur \\ CH-8401 \\ SWITZERLAND \\ +41522677148 \\ dominic.bechaz@zhwin.ch
}

Mr. Gary Bernhardt

VXI Technology Inc.

5425 Warner Rd, Ste 13

Valley View, OH 44125

USA

216-233-3799

garyb@vxitech.com

\section{Mr. Antonius Boller}

Siemens AG, A\&D PT 2 M

P.O. Box 4848

Nuremberg, 90327

GERMANY

+49 (911) 895-5071

+49 (911) 895-4845

antonius.boller@siemens.com

Mr. Tan Boon Keat

Agilent Technologies

Bayan Lepas, 11900

MALAYSIA

604-680-5744

gilbert-bk_tan@agilent.com

Bruce Boyes

Systronix Inc

555 S 300 East

Salt Lake City, UT 84111

USA

801-809-2658

801-534-1019

bboyes@systronix.com

\section{Mr. Jim Brad}

USNO

3450 Mass Ave., NW

Washington, DC 20392

USA

202-762-1452

brad.jim@usno.navy.mil

Seela Raj D. Rajaiah

Agilent Technologies

Bayan Lepsa FIZ

Bayan Lepas 11900

MALAYSIA

604-680-8741

seela-raj_raj@agilent.com 


\author{
Dr. Patrick Diamond \\ Semtech Corp \\ 200 Flynn Rd \\ Camarillo, CA 93012 \\ USA \\ 805-469-3530 \\ pdiamond@semtech.com

\section{Dr. John Eidson} \\ Agilent Technologies \\ 3500 Deer Creek Rd, MS 4-M-A \\ Palo Alto, CA 94303 \\ USA \\ 650-485-4263 \\ 650-485-3894 \\ john_eidson@agilent.com

\section{Mr. Mark Elliot} \\ Symmetricom \\ 3750 Westwind Blvd \\ Santa Rosa, CA 95403 \\ USA \\ 707-636-1932 \\ 707-527-6640 \\ melliot@symmetricom.com

\section{Tom W. Farley} \\ Symmetricom \\ 100 Michael Angelo Way \\ Bldg E, Ste 900 \\ Austin, TX 78728-1257 \\ USA \\ 512-721-4058 \\ 512- 721-4603 \\ tfarley@symmetricom.com

\section{Mr. John Fischer} \\ Spectracom Corp. \\ 95 Methodist Hill Dr., Ste 500 \\ Rochester, NY 14623 \\ USA \\ 585-321-5800 ext. 112 \\ jfischer@spectracomcorp.com \\ Mr. Douglas Fowley \\ GE Energy \\ 1501 Roanoke Blvd, Rm 281 \\ Salem, VA 24153 \\ USA \\ 540-387-8598 \\ 540-387-8651 \\ Douglas.Fowley@ge.com
}

\author{
Mr. Michael Gerstenberger \\ KUKA Development Labs \\ 22500 Key Dr \\ Clinton Township, MI 48360 \\ USA \\ 248-844-0561 \\ 248-844-0505 \\ MichaelGerstenberger@KukaDevLabs.com
}

\section{Mr. James Gilsinn}

NIST

100 Bureau Dr, MS 8230

Gaithersburg, MD 20899-8230

USA

301-975-3865

301-990-9688

\section{Mr. Tarrance Graham}

Weed Instrument Company

707 Jeffrey Way

Round Rock, TX 78664

USA

512-434-2817

512-434-2851

tgraham@weedinstrument.com

\section{Dr. John Guilford}

Agilent Technologies

1615 75th St SW

Everett, WA 98203

USA

425-356-6057

\section{Ken Harris}

Rockwell Automation

1 Allen-Bradley Dr

Mayfield Heights, OH 44124

USA

440-646-3621

krharris@ra.rockwell.com

\section{Mr. Oyvind Holmeide}

OnTime Networks AS

Glads vei 20

Oslo, 489

NORWAY

$+4722090304$

$+4722090310$

oeyvind@ontimenet.com 


\author{
Hassan Hussain \\ Agilent Technologies \\ Bayan Lepas FTZ \\ Penang, 11900 \\ MALAYSIA \\ +604-6805523 \\ hassan_hussain@agilent.com \\ Dominic Iadonis \\ Hirschmann \\ 20440 Century Blvd \\ Germantown, MD 20874 \\ USA \\ 240-686-2329 \\ diadonisi@hirschmann-usa.com \\ Mr. Nikolaus Kero \\ Oregano Systems Ltd. \\ Phorusgasse 8 \\ Vienna, A-1040 \\ AUSTRIA \\ +436763126964 \\ +43676 8431 04-888 \\ keroe@oregano.at \\ Mr. Robert Kroboth \\ Agilent Technologies \\ 1900 Garden of the Gods Rd \\ Colorado Springs, CO 80919 \\ USA \\ 719-531-4415 \\ bob_kroboth@agilent.com \\ Mr. Kang Lee \\ NIST \\ 100 Bureau Dr., Stop 8220 \\ Gaithersburg, MD 20899-8220 \\ USA \\ 301-975-6604 \\ 301-990-3851 \\ kang.lee@nist.gov

\section{Ms. Ya-Shian Li \\ NIST} \\ 100 Bureau Dr \\ Bldg. 225, Room A47 \\ Gaithersburg, MD 20899 \\ USA \\ 301-975-5319 \\ 301-975-8069 \\ ya-shian.li@nist.gov
}

\author{
Mr. Mondy Lim \\ Semtech \\ 10 Wimbledon Way \\ Ottawa K2K3J2 \\ CANADA \\ 613-862-1188 \\ mlim@semtech.com

\section{Mr. Hung Mach} \\ Boeing \\ P.O. Box 3707 \\ MS 14-ME \\ Seattle, WA 98124 \\ USA \\ 206-655-9926 \\ 206-655-7724 \\ hung.k.mach@boeing.com \\ Mr. John Mackay \\ Progeny Systems \\ 9500 Innovation Dr \\ Manassas, VA 20110 \\ USA \\ 703-368-6107 ext. 144 \\ 703-331-5651 \\ john.mackay@progeny.net
}

\section{Dan Maxwell}

Tarallax Wireless Inc

10 W 100 S, Ste 600

Salt Lake City, UT 84101

USA

801-533-2727

801-355-0289

dmaxwell@tarallax.com

Mr. Matthew McConnell

VXI Technology, Inc

5425 Warner Rd, Ste 13

Valley View, OH 44125

USA

216-447-4059

mattm@vxitech.com

Mr. Paul Mecklenburg

VXI Technology, Inc

5425 Warner Rd, Ste 13

Valley View, OH 44125

USA

216-447-4059

216-447-4059

paulm@vxitech.com 


\author{
Mr. Dirk Mohl \\ Hirschmann Electronics \\ Stuttgarter Strasse 45-51 \\ Neckartenzlingen, 72654 \\ GERMANY \\ dmohl@nt.hirschmann.de \\ Mr. Anatoly Moldovansky
Rockwell Automation
1 Allen-Bradley Dr
Mayfield Hts., OH 44124
USA
440-646-5369
440-646-3076
amoldovansky@ra.rockwell.com \\ David Moyer \\ Semtech Corp \\ 958 Spring City Rd \\ Phoenixville, PA 19460 \\ USA \\ 610-792-9395 \\ dmoyer@semtech.com \\ Mr. Paul Myers \\ Spectracom Corp. \\ 95 Methodist Hill Dr., Ste 500 \\ Rochester, NY 14623 \\ USA \\ 585-321-5800 ext. 158 \\ pmyers@spectracomcorp.com

\section{Dr. Lee Ng} \\ Agilent Technologies Inc. \\ 3500 Deer Creek Rd \\ Palo Alto, CA 94304 \\ USA \\ 650-485-5726 \\ lee_ng@agilent.com

\section{Mr. Sven Nylund} \\ OnTime Networks AS \\ Glads vei 20 \\ Oslo, 489 \\ NORWAY \\ $+4722090308$ \\ 4722090310 \\ sven@ontimenet.com
}

\author{
Ms. Karen O'Donoghue \\ NSWCDD \\ 17320 Dahlgren Rd \\ Dahlgren, VA 22448 \\ USA \\ 540-653-1567 \\ 540-653-8673 \\ odonoghuekf@nswc.navy.mil
}

Afshaneh Pakdaman

P.O. Box 2202

Santa Clara , CA 95055

USA

afshan@sfsu.edu

\section{Mr. Rick Pennavaria}

Weed Instrument Co

P.O. Box 300

Round Rock, TX 78680

USA

512-434-2917

\section{Edward Powers}

3450 Mass Ave., NW

Washington, DC 20392

USA

202-762-1451

202-762-1511

powers.edward@usno.navy.mil

Mrs. Silvana Rodrigues

Zarlink Semiconductor

400 March Rd

Ottawa, K2K 3H4

CANADA

613-270-7590

613-592-1010

silvana.rodrigues@zarlink.com

\section{Mr. Michael Rupert}

Zarlink Semiconductor 400 March Rd

Ottawa, K2K 3H4

CANADA

613-592-0200 ext. 7347

michael.rupert@zarlink.com 


\author{
Mr. Paul Rushton \\ Semtech Corp. \\ Units 2 \& 3 Park Court \\ Premier Way \\ Hampshire, S051 9DN \\ UNITED KINGDOM \\ 4401794527600 \\ prushton@semtech.com

\section{Mr. Stephan Schuler} \\ SIEMENS AG \\ Brauckstr. 14 \\ Witten, 58449 \\ GERMANY \\ +4923026672063 \\ +4923026673228 \\ schueler.stephan@siemens.com

\section{Mr. Puneet Sharma} \\ Intel Corp \\ 5000 W Chandler Blvd \\ Chandler, AZ 85226 \\ USA \\ puneet.sharma@intel.com

\section{Dr. Mark Shepard} \\ GE Energy \\ 1501 Roanoke Blvd \\ Salem, VA 24153 \\ USA \\ 540-387-8710 \\ mark.e.shepard@ge.com

\section{Mr. Paul Skoog} \\ Symmetricom \\ 3750 Westwind Blvd \\ Santa Rosa, CA 95403 \\ USA \\ 707-636-1955 \\ pskoog@symmetricom.com

\section{Yuyin Song} \\ NIST \\ 100 Bureau Drive, MS 8220 \\ Gaithersburg, MD 20899 \\ USA \\ 301-975-6542 \\ yuyin.song@nist.gov
}

\author{
Samuel Stein \\ Timing Solutions Corp \\ 4775 Walnut St, Ste 1B \\ Boulder, CO 80301 \\ USA \\ 303-939-8481 \\ 303-443-5152 \\ lfischer@timing.com

\section{John Stratton} \\ Agilent Technologies \\ 1400 Fountaingrove Pkwy \\ Bldg 3LS-V \\ Santa Rosa, CA 95403 \\ USA \\ 707-577-3838 \\ 707-577-5834 \\ john_stratton@agilent.com
}

\author{
Mr. David Tonks \\ Semtech Corp \\ Units 2 \& 3 Park Ct \\ Primier Way \\ Hampshire, S051 9DN \\ UNITED KINGDOM \\ + 441974529600 \\ dtonks@semtech.com \\ Mr. Murray Tysinger \\ Agilent Technologies \\ 5301 Stevens Creek Blvd \\ MS-51U-23 \\ Sants Clara,CA 95051 \\ USA \\ 408-553-2640 \\ 408-553-6591 \\ butch_tysinger@agilent.com \\ Dr. Bradley Van Eck \\ Intl SEMATECH \\ 2706 Montopolis Dr \\ Austin, TX 78741 \\ USA \\ 512-356-3981 \\ 512-356-7848 \\ brad.van.eck@ismi.sematech.org
}


Mr. Saso Vlahu

ST Microelectronics

1300 E Woodfield Rd, Ste 410

Schaumburg, IL 60173

USA

847-585-3017

847-517-1943

saso.vlahu@st.com

Dr. Aljosa Vrancic

National Instruments

11500 N Mopac Expwy

Austin, TX 78759

USA

512-683-8082

aljosa.vrancic@ni.com

\section{Dr. Karl Weber}

Siemens

Wuerzburger Str. 121

Fuerth, 90766

GERMANY

+491712307569

karl.weber@siemens.com

\section{Mr. Hans Weibel}

Zurich Univ of Applied Sciences

Technikumstrasse 9

Postfach 805

Winterthur, CH-8401

SWITZERLAND

+41522677552

hans.weibel@zhwin.ch
Dr. Joseph White

U.S. Naval Research Lab

4555 Overlook Ave SW

Code 8150

Washington, DC 20375

USA

202-767-5111

202-767-4050

joe.white@nrl.navy.mil

\section{Mr. Michael Wilson}

Agilent

14040 Pierce Rd

Saratoga, CA 95070

USA

408-867-5526

mikewilson30@comcast.net

\section{Steven Wissler}

1322 Marie Ave

Ephrata, PA 17522

USA

717-393-3831 ext. 140

stevew@godfrey.com

Mr. Steven Zuponcic

Rockwell Automation

1 Allen-Bradely Dr

Mayfield Hts., OH 44124

USA

440-646-5384

440-646-4344 